\title{
Estudio sobre actitudes frente a la ciencia de los estudiantes del internado rotatorio de la carrera de medicina en la Universidad de San Francisco Xavier de Chuquisaca
}

\author{
Ivonne F. Ramírez-Martínez, César Maldonado-Sanabria, Richar Villacorta-Guzmán, Germán Gallardo-Matienzo
}

Objetivo. Analizar la imagen de la ciencia y la enseñanza de la ciencia en estudiantes universitarios del internado rotatorio en medicina de la Universidad de San Francisco Xavier de Chuquisaca en Sucre, Bolivia.

Sujetos y métodos. El estudio se realizó en 101 estudiantes universitarios del internado rotatorio intrahospitalario del municipio de Sucre, Bolivia. Se aplicó un cuestionario que valora las actitudes frente a la ciencia, es decir, la imagen y la enseñanza de la ciencia en su formación universitaria.

Resultados. Se encontró que la imagen y la enseñanza de la ciencia en estudiantes del internado de medicina están por encima de la media esperada, aunque la praxis sea aún incipiente, como se observa por la baja participación en proyectos y publicaciones locales.

Conclusiones. Las actitudes frente a la ciencia son positivas, pero el reto para la formación universitaria será aplicar las políticas de desarrollo de investigación en salud, si se busca incidir en la mejora de los indicadores sociosanitarios del país.

Palabras clave. Actitudes. Ciencia. Enseñanza de la ciencia. Internado rotatorio. Investigación. Medicina.

\section{Research regarding attitudes towards sciences at San Francisco Xavier of Chuquisaca University on medicine students doing internships}

Aim. To analyse the image of science, the teaching of it that medicine students, doing their internships at the San Francisco Xavier of Chuquisaca University, have.

Subjects and methods. The research was made among 101 medicine students that are doing their internships at the hospital system in Sucre, Bolivia. A survey that values the attitudes towards science was applied to them; this is, how they value the image of science and how science is taught throughout their university education.

Results. It was found that the attitudes among medicine students doing their internships, towards science, are positive; however the praxis of it is still minimal, due to the incipient participations in research projects and publications.

Conclusions. The attitudes towards science are positive, however the challenge will be, for the university, to apply and develop the health research policies, this way the social-sanitary indicators will be improved.

Key words. Attitudes towards science. Medicine. Medicine internship. Research. Science.

\section{Introducción}

Debido a la importancia que ha cobrado la ciencia y la tecnología como herramienta estratégica para el desarrollo, las instituciones educativas que forman a los futuros profesionales deberán trabajar en sus aulas para aportar y construir un mejor futuro para el país. Los educadores debemos comprometernos con ese reto y trabajar desde los niveles escolares en esta tarea. Aún quedan muchos desafíos que los educadores debemos afrontar, los desafíos deben empezar por conocer cuáles son las actitudes de los educandos frente a la ciencia médica y en qué medida están involucrados con la solución de las problemáticas de la salud del hoy y del mañana.

Algunos indicadores en Bolivia presentan temas de vital importancia en salud, y muestran que la mortalidad materna alcanza a 290/100.000; la tasa de mortalidad infantil es de 50/1.000; la tasa de mortalidad neonatal es de 25/1.000; y los nacimientos con alguna discapacidad son 15/1.000. En cuanto a la población con virus de la inmunodeficiencia humana (VIH)/sida entre las edades de 15-49 años, se tienen datos de afectación del 0,2\% [1].
Universidad Mayor, Real y Pontificia de San Francisco Xavier de Chuquisaca. Sucre, Bolivia.

Correspondencia: Dra. Ivonne Fabiana Ramírez Martínez. Comité Académico Doctoral. Universidad Mayor, Real y Pontificia de San Francisco Xavier de Chuquisaca. San Alberto, 204. Casilla Postal 981. Sucre, Bolivia.

E-mail: ifrm14@gmail.com Recibido: 15.11.16.

Aceptado: 07.03.17.

Conflicto de intereses: No declarado.

Competing interests: None declared.

(C) 2017 FEM 
Según el informe de la ENDSA [2], cerca del 90\% de los hombres y el $85 \%$ de las mujeres, adolescentes y jóvenes, tienen información sobre el VIH y el sida, pero no hay datos sobre el conocimiento de otras infecciones de transmisión sexual que también afectan a la salud en esta etapa del desarrollo humano. Este informe señala que alrededor del $76 \%$ de los hombres y el $64 \%$ de las mujeres, entre 15 a 24 años, tienen conocimiento de métodos para reducir el riesgo de contraer el VIH. Estos y otros temas priorizados por la agenda de salud del país reflejan la necesidad de que la universidad asuma un compromiso de enseñanza y de que los futuros profesionales contribuyan, a través de la investigación, a mejorar los indicadores actuales de salud en el país.

Se tienen muchos antecedentes [3,4] que documentan la medición de las actitudes frente a la ciencia que adaptan el proyecto ROSE [5], con investigaciones realizadas en el contexto educativo escolar y que se constituyen en referentes de esta investigación.

Los estudios realizados sobre actitudes de las nuevas generaciones frente a la ciencia y la tecnología, en contextos fuera de Bolivia, no son los más positivos [6]. Datos muy parecidos se encuentran en otros estudios similares, como en Croacia [7], donde realizaron un estudio con 932 estudiantes universitarios de medicina. La tasa de respuesta fue del $58 \%$. Obtuvieron una media de conocimiento pobre de 3,2 $\pm 1,7$ con ocho preguntas realizadas, y la media de actitud fue de 3,68 en la escala de Likert, cuyo valor máximo es 5 .

Amin et al [8] realizaron un estudio similar en tres universidades árabes con la participación de 423 estudiantes. Encontraron una media de conocimiento de $3,6 \pm 1,7$. Un $69 \%$ de los estudiantes mostraba una actitud positiva hacia la investigación.

El estudio de Khan et al [9] con estudiantes de medicina de Pakistán encontró una media de conocimiento de 49 de un total de 100 puntos, lo que se consideró como un conocimiento moderado.

Por otro lado, los estudiantes de medicina de la Universidad de Panamá tienen un pobre conocimiento y una actitud regular hacia la investigación científica, y a medida que avanzan en sus estudios aumenta el bagaje de conocimiento, pero disminuye la actitud hacia la investigación científica [10].

Asimismo, un estudio realizado por otros autores [11] muestra un punto muy débil en la enseñanza de la ciencia, y destaca que la educación científica debería plantearse con seriedad en lo referente a la educación sobre actitudes relacionadas con la ciencia. En esa misma línea, Solaz et al [12] consiguieron mejorar las actitudes ante la ciencia tras la aplicación de una metodología constructivista en profesores de educación primaria de la Universidad de Valencia, lo que muestra la importancia de la influencia del proceso educativo.

En estudios anteriores a bachilleres de colegios de la ciudad de Sucre se encontraron resultados similares, es decir, que la escuela no desempeña un papel importante en la educación científica de estos jóvenes, ni fomenta las actitudes ni las vocaciones científicas, a pesar del optimismo en lo que pueda hacer y lograr la ciencia [13].

Esto supone planificar la enseñanza de la ciencia en el currículo, trabajarla en el aula $[11,14]$; se debe, por tanto, repensar la forma de aproximar a los estudiantes de secundaria, desde la formación y para la motivación hacia la ciencia. Estas conclusiones fueron extraídas de estudios anteriores [14].

En las últimas décadas, la investigación sobre concepciones alternativas ha reiterado que los estudiantes fracasan en aprender significativamente los contenidos cognitivos y conceptuales en ciencias [15]. Rocard et al [11] señalan que no resulta extraño el rechazo a la ciencia como carrera y profesión, y esta constatación causa el bajo interés científico.

Los jóvenes de secundaria con intereses científicos muestran una actitud positiva hacia la ciencia $[6,16]$. Estos grupos también tienen una actitud positiva respecto al medio ambiente.

Otros estudios muestran que existe un rechazo claro a la posibilidad futura de realizar trabajos en ciencia y tecnología $[17,18]$. Esto ratifica que se reconoce la importancia de la ciencia sin inclinarse a ser partícipes de ella.

Por otra parte, un estudio sobre actitud hacia la ciencia en estudiantes de la salud realizado por Plazas et al [19] concluye lo siguiente: la actitud favorable de los estudiantes puede ser explicada por el contacto temprano con el método científico en su carrera. El valor y el dominio de la metodología científica fueron los menos valorados por los encuestados, y podría relacionarse con su desconocimiento. Estas conclusiones apoyan lo señalado por Domínguez [20], quien encontró que la imagen de la ciencia es errónea en estudiantes universitarios, lo que influye en sus actitudes frente a ésta.

Una investigación realizada sobre la producción científica de los estudiantes universitarios de medicina en el Perú [21] muestra que es escasa por distintos motivos: se destaca la falta de infraestructura, cuyo coste económico es alto, por lo que frecuentemente la universidad lo relega para dar más importancia a la docencia; la carga académica de la mayoría de los profesores y de alumnos de tiempo completo es grande; el acceso a redes de comunicación resulta muy limitado y específico para algunas 
áreas; la vinculación docencia-investigación es escasa y con frecuencia difícil de desarrollar. Además, numerosos profesores de carrera no cuentan con una formación adecuada en el área de investigación, y en su mayoría no existe una cultura de publicación de los resultados que se obtienen de las investigaciones, sea por el temor a la crítica o por la poca habilidad para escribir;. Por ello se dice que las actitudes no repercuten en la práctica de investigación.

Sobre la variable sexo, las revisiones muestran que la actitud hacia la ciencia no se diferencia entre hombres y mujeres. La edad tampoco es una variable que evidencia diferencias. Estos resultados coinciden con los hallazgos de otros estudios [7].

En contraposición a los anteriores, son los casos árabe [8] y pakistaní [9] los que mostraron que los estudiantes de medicina tienen actitudes positivas respecto a la ciencia y que su imagen es también relativamente positiva.

Por los antecedentes revisados se puede decir que las universidades deberán formar a las futuras generaciones de científicos para mejorar la calidad de vida en los países. Los resultados de investigaciones iniciales indican que los centros de educación superior tienden a ahogar las ilusiones y vocaciones científicas en vez de continuarlas e impulsarlas [13].

\section{Sujetos y métodos}

El estudio es descriptivo, de tipo cualicuantitativo, realizado en los centros hospitalarios de los distritos 1 y 2 del municipio de Sucre, Bolivia. La muestra fue de 101 estudiantes de internado rotatorio de la Facultad de Medicina que realizan sus prácticas preprofesionales en hospitales de segundo y tercer nivel del municipio indicado.

El internado rotatorio es parte del plan de estudios, previo a la titulación, y se realiza en el sexto año de la formación universitaria. El objetivo es que el estudiante o interno efectúe rotaciones entre los diferentes servicios hospitalarios con el fin de ir adquiriendo conocimientos, habilidades y valores propios de cada especialidad médica. La muestra estuvo conformada por 101 estudiantes, 48 universitarios y 53 universitarias. Previamente a la aplicación de la encuesta, los universitarios firmaron el consentimiento informado para participar del estudio.

Para la recogida de datos, como en estudios anteriores [13], se procedió a aplicar el cuestionario de Vázquez y Manassero [3], adaptado del proyecto ROSE [5]. Contiene 24 ítems que evalúan aspectos relacionados con la ciencia mediante una escala Likert con criterios del 1 al 4.

\begin{tabular}{|c|c|c|c|}
\hline & & $\begin{array}{l}\text { Imagen de } \\
\text { la ciencia }\end{array}$ & $\begin{array}{c}\text { Ciencia } \\
\text { universitaria }\end{array}$ \\
\hline \multirow{3}{*}{$\begin{array}{l}\text { Sexo } \\
\text { masculino }\end{array}$} & $n$ & 48 & 48 \\
\hline & Media & 22,9167 & 45,4583 \\
\hline & Desviación estándar & 2,87937 & 5,61517 \\
\hline \multirow{3}{*}{$\begin{array}{l}\text { Sexo } \\
\text { femenino }\end{array}$} & $n$ & 53 & 53 \\
\hline & Media & 22,6226 & 45,4151 \\
\hline & Desviación estándar & 3,3697 & 7,45611 \\
\hline \multirow{3}{*}{ Total } & $n$ & 101 & 101 \\
\hline & Media & 22,7624 & 45,4356 \\
\hline & Desviación estándar & 3,13416 & 6,61274 \\
\hline
\end{tabular}

El contenido de las cuestiones seleccionadas se centra en tres escalas: siete sobre la imagen de la ciencia, 15 sobre actitudes hacia la ciencia escolar y dos sobre actitudes hacia el medio ambiente; este último no formó parte del análisis realizado. Para el estudio del caso de medicina se tomaron en cuenta los reactivos sobre imagen y actitudes en la enseñanza de la ciencia.

Los resultados de la imagen de la ciencia y la enseñanza de la ciencia fueron analizados basándose en los criterios de 1 al 4, donde 2,5 es la media teórica, como refieren estudios anteriores realizados por Vázquez y Manassero [4].

\section{Resultados}

El procesamiento de los datos muestra importantes aspectos que hay que considerar sobre las dos categorías, imagen de la ciencia y enseñanza de la ciencia en la universidad.

A continuación se muestran los valores del $\alpha$ de Cronbach para las dimensiones imagen de la ciencia $(0,7)$ y enseñanza de la ciencia universitaria $(0,8)$; por tanto, tienen una fiabilidad superior a 0,5 en ambos casos, lo que muestra la consistencia interna de la escala.

Como se evidencia en la tabla I, no existen diferencias estadísticamente significativas entre las medias aritméticas de varones y mujeres en las dimensiones de imagen de la ciencia y enseñanza de la ciencia. 
Tabla II. Correlaciones entre imagen de la ciencia y ciencia universitaria.

\begin{tabular}{|c|c|c|c|}
\hline & & $\begin{array}{l}\text { Imagen de } \\
\text { la ciencia }\end{array}$ & $\begin{array}{c}\text { Ciencia } \\
\text { universitaria }\end{array}$ \\
\hline \multirow{3}{*}{$\begin{array}{l}\text { Imagen de } \\
\text { la ciencia }\end{array}$} & Correlación de Pearson & 1 & $0,349^{a}$ \\
\hline & Sig. (bilateral) & & 0 \\
\hline & $n$ & 101 & 101 \\
\hline \multirow{3}{*}{$\begin{array}{l}\text { Ciencia } \\
\text { universitaria }\end{array}$} & Correlación de Pearson & $0,349^{a}$ & 1 \\
\hline & Sig. (bilateral) & 0 & \\
\hline & $n$ & 101 & 101 \\
\hline \multirow{3}{*}{$\begin{array}{l}\text { Medio } \\
\text { ambiente }\end{array}$} & Correlación de Pearson & $0,519^{a}$ & $0,327^{a}$ \\
\hline & Sig. (bilateral) & 0 & 0,001 \\
\hline & $n$ & 101 & 101 \\
\hline
\end{tabular}

a Correlación significativa en el nivel 0,01 (dos colas).

Para determinar si la actitud de los estudiantes es favorable, se dividieron los valores de las medias de cada dimensión por su respectivo número de ítems y se contrastaron con el valor de la media teórica de 2,5 , y se obtuvo en imagen de la ciencia un valor de 3,2 y, en cuanto a la enseñanza de la ciencia universitaria, un valor de 3; como ambos valores están por encima de la media teórica, se concluye que los estudiantes tienen una actitud favorable en ambas dimensiones de las actitudes frente a la ciencia.

La tabla I muestra similares resultados en los grupos femenino y masculino, tanto en la dimensión imagen de la ciencia como en el caso de la dimensión enseñanza de la ciencia.

En la tabla II se observa que existe relación lineal significativa de 0,01 entre las dos dimensiones objeto de estudio, es decir, que quienes tienen una imagen positiva de la ciencia coinciden con un concepto adecuado de la enseñanza de la ciencia en su formación universitaria.

Para complementar el estudio se realizó un análisis documental de las publicaciones de las bases de datos de la Universidad de San Francisco Xavier de Chuquisaca durante las tres últimas gestiones, donde no se encontraron artículos publicados por estudiantes referidos a la ciencia médica. En la gestión académica 2015 se presentaron 22 proyectos de investigación a las jornadas científicas internas en las distintas áreas de la salud, de los cuales ninguno correspondía al área médica; por otra parte, tampoco se tienen publicaciones en la Revista de Ciencia, Tecnología e Innovación [22].

Las memorias de la I y II Feria Nacional de Ciencia y Tecnología 2015 (La Paz) y 2016 (Sucre) no documentan ningún trabajo finalista en medicina por parte de la Universidad de San Francisco Xavier de Chuquisaca [23,24].

La Sociedad Científica de Medicina, que participa en eventos de actualidad médica, tiene como miembros a estudiantes de segundo a último año. Los internos, debido a la alta actividad intrahospitalaria de turnos de 10 horas/día, no participan de ellas y carecen de publicaciones, sobre todo porque su práctica tiene un alto componente clinicoasistencial y poco investigativo. La revista publicada por esta sociedad tiene baja producción, lo que puede deberse principalmente al perfil clinicoasistencial predominante en esta etapa de la formación en la carrera.

En la rotación de salud comunitaria, los internos realizan tres meses de prácticas en comunidades dispersas de los municipios del departamento. La producción final de esa rotación es la notificación de una investigación relacionada con las problemáticas de salud pública, pero se quedan en ejercicios académicos que sólo son documentados para cumplir un requisito y quedar en los archivos físicos de la biblioteca facultativa.

La modalidad de graduación única es el internado rotatorio, lo que limita la posibilidad de formar al futuro médico con un perfil científico y de investigación.

\section{Discusión}

Las conclusiones de los estudios de autores como Alonso [4] podrían discutirse en el estudio realizado, pues las actitudes de los estudiantes del internado de medicina son buenas, están por encima de la media esperada, a diferencia de los resultados encontrados en universidades peruanas [25].

A los residentes médicos, la ciencia les parece útil e importante. El reto será trabajar para que ellos la practiquen, pues su participación actual en equipos, redes y proyectos de investigación es baja o nula.

Estudios previos señalan que la imagen de la ciencia no es baja, como los de Vázquez y Manassero [3] y Carrillo-Larco y Carnero [15]. Éste es un punto a favor y un desafío para la educación en ciencia, por lo que la universidad debe motivar y desarrollar las actitudes, como señalan otros estudios [25] de casos revisados en carreras médicas en las universidades del Perú. 
Sobre la enseñanza de la ciencia, Rocard et al [11] indican que la educación científica debería plantearse con seriedad. Para otros estudiosos supone planificarla en el currículo, trabajarla en el aula [13]. Desde esta perspectiva, el estudio ratifica que, como hay buenas actitudes frente a la ciencia en el grupo de residentes, sólo queda planificar mejor el currículo para subir y mantener esos resultados. De este modo, la imagen y las actitudes positivas respecto a la ciencia no sólo serán expectativas o ideales, sino que serán realidades obradas.

En los estudios de Amin et al [8] y Khan et al [9] se observan diferencias con el caso estudiado, pues ellos, en imagen y enseñanza de la ciencia, son ligeramente menos optimistas. Estos datos guardan relación con la constante de que las actitudes, imagen respecto a la ciencia y enseñanza de la ciencia, son positivas, por lo menos en el sentido ideal. Asimismo, en el estudio realizado en San Francisco con los internos de medicina, no se observaron diferencias en las actitudes frente a la ciencia entre las mujeres y los varones, como ya señalaron Hren et al [7].

El caso de la universidad panameña [10] es más pesimista. Los estudiantes son menos optimistas en el momento de considerar las imágenes de la ciencia y las actitudes respecto a ella. En países como Bolivia, el estatus del médico desempeña un papel importante en el momento de definir un perfil profesional; a esto se suma la problemática laboral, puesto que el Ministerio de Salud y Deportes en Bolivia tiene su organigrama basado en la medicina asistencialista. Los laboratorios y centros de investigación en Bolivia apenas tienen 10 personas contratadas; esto muestra las limitaciones para la promoción de la investigación en los médicos. No hay inversión ni promoción de recursos humanos en investigación en salud.

El equipamiento en Chuquisaca no llega a satisfacer las necesidades básicas, lo que también influye en la baja o nula producción académica; al no contar con la implementación necesaria, la investigación es de tendencia negativa.

De los 344 centros e institutos de investigación, estatales y privados, en Bolivia, sólo 31 se encuentran en el departamento de Chuquisaca, lo que representa sólo el 9\% del total. Veintisiete de estos institutos son de medicina clínica, dos de medicina básica y tres de ciencias de la salud [25].

El caso de las universidades peruanas $[20,25,26]$ muestra similares casos a los de las universidades panameñas; el caso peruano constata que la cultura científica es escasa, a lo que se puede sumar el poco presupuesto destinado a la investigación; el caso es similar en Bolivia.
La experiencia peruana es coincidente con la realidad de la Universidad Boliviana, lugar desde el que se crea un imaginario de acercamiento a la ciencia; empero, las condiciones de investigación, publicación y, ante todo, de inserción en el ámbito académico tienen enunciado propagandístico, con poca o ninguna efectividad en el quehacer académico.

Siguiendo a Díaz-Vélez et al [21], el peso de la academia recae en la actividad docente reproductiva, que no vincula la docencia-investigación; asimismo, se percibe que los profesores de carrera no integran redes de investigación, ni cuentan con publicaciones de impacto que documenten las investigaciones realizadas. La carga docente sólo se reduce a la transmisión de conocimientos, no hay producción de conocimientos ni interacción social. Estas carencias perjudican y casi extinguen el inicial optimismo encontrado en los internos de medicina respecto a la imagen, los intereses y la enseñanza de la ciencia.

Las actitudes frente a la ciencia en estudiantes del internado rotatorio de medicina son positivas; pero este optimismo resulta contradictorio, pues existe reconocimiento de que la ciencia contribuye a la transformación social; luego no se observa alto interés ni participación activa en eventos locales, nacionales e internacionales. La producción científica es escasa y, a menudo, nula. El aspecto de la salud de la Agenda Patriótica del Estado Plurinacional de Bolivia sigue estando desatendido por el Estado y las universidades que forman en salud.

En conclusión, la imagen de la ciencia en el grupo estudiado es positiva, lo que en esta etapa del desarrollo de los estudiantes del internado rotatorio de medicina resulta favorable; sin embargo, el desafío será desarrollar esos intereses científicos en pro de que se involucren en procesos científicos con temas prioritarios en salud.

Se observa un divorcio entre los intereses de los estudiantes de medicina por la ciencia y la debilidad en la formación científica. Este divorcio tiene como consecuencia la declinación de las actitudes científicas de los jóvenes a medida que avanzan hacia niveles superiores; la poca motivación y la nula conformación de redes de investigadores que los sostengan podrían disminuir el alto grado de interés de los estudiantes por la ciencia.

En el caso estudiado, la carrera de medicina no promueve ni incentiva lo suficiente a la formación científica. Los futuros médicos reconocen la importancia de la ciencia, lo cual se tiene como un incentivo pasajero, debido a que el hecho de dedicarse a la repetición, memorización y aprobación del nivel res- 
pectivo influye para que su participación y aporte con producciones locales sean deficitarios, situación que se prolonga en el ámbito profesional, institucional y de salud, en general. Esta situación no permite la relación de conocimientos adquiridos direccionados hacia los conocimientos potenciales con los cuales egresan los médicos de las universidades.

No existen diferencias significativas de sexo respecto a las actitudes, interés, imagen y enseñanza de la ciencia universitaria. La similitud que alcanza a las percepciones sobre la ciencia es positiva.

El sistema universitario de la Universidad Mayor Real y Pontificia de San Francisco Xavier de Chuquisaca y el Sistema Nacional de Salud deben repensar, adecuar e implementar sus políticas de ciencia, tecnología e innovación para promover las vocaciones científicas y captar a futuros médicos investigadores que se involucren en procesos de investigación contribuyendo a elevar los indicadores de salud pública y colectiva en el Estado.

La universidad tiene un papel importante, dado que hay buena imagen sobre la ciencia en estudiantes de medicina en el internado rotatorio; queda incentivar, motivar y acompañar la formación científica de quienes pueden, potencialmente, dedicar su carrera profesional a la producción científica. Es fundamental impulsar y continuar los intereses científicos enmarcados en el aporte al desarrollo teórico de nuestra realidad en salud.

\section{Bibliografía}

1. Population Reference Bureau. Población y desarrollo económico. Cuadro de datos. URL: http://www.prb.org/pdf12/populationeconomic-development-2012_sp.pdf.

2. Instituto Nacional de Estadística. encuesta nacional de demografía y salud 2008. URL: http://es.scribd.com/doc/ 22108642/Encuesta-Nacional-de-Demografia-y-SaludENDSA-2008.

3. Vázquez A, Manassero MA. La relevancia de la educación científica. Palma de Mallorca: Servei de Publicacions de la Universitat de les Illes Balears. Conselleria d'Economia, Hisenda i Innovació; 2007.

4. Alonso TJ. Diseño y validación de un instrumento para medir la formación científica en estudiantes de enfermería [tesis doctoral]. México: UNAM; 2014.

5. Schreiner C, Sjøberg S. Sowing the seeds of ROSE. Background, rationale, questionnaire, development, and data collection for ROSE. The relevance of science education. A comparative study of students' views of science and science education. Acta Didactica 4. Oslo: University of Oslo; 2004. URL: http:// www.ils.uio.no/forskning/rose/documents/AD0404.pdf.

6. Vázquez A, Manassero MA. La relevancia de la educación científica: actitudes y valores de los estudiantes relacionados con la ciencia y la tecnología. Revista de Investigación y Experiencias Didácticas 2009; 27: 33-48.

7. Hren D, Lukic IK, Marusic A, Vodopivec I, Vujaklija A, Hrabak M, et al. Teaching research methodology in medical schools: students' attitudes towards and knowledge about science. Med Educ 2004; 38: 81-6.
8. Amin T, Kaliyadan F, Al Gattan E. Knowledge, attitudes and barriers related to participation of medical students in research in three Arab universities. Educ Med J 2012; 4: 43-56.

9. Khan H, Khan S, Iqbal A. Knowledge, attitudes and practices around health research: the perspective of physicians-intraining in Pakistan. BMC Medl Educ 2009; 9: 46-54.

10. Silva S, Zúñiga l, Ortega C, Yau A, Castro F, Barría JM, et al. Conocimientos y actitudes acerca de la investigación científica en los estudiantes de medicina de la Universidad de Panamá. Archivos de Medicina 2013; doi: 10.3823/1200.

11. Rocard M, Csermely P, Jorde D, Lenzen D, Walberg-Henriksson $\mathrm{H}$, Hemmo V. Science education now: a renewed pedagogy for the future of Europe. Brussels: European Commission; 2007.

12. Solaz J, Vicent SJ, Caurin C. Cambio en las actitudes hacia el estudio de las ciencias en alumnos universitarios: efectos de usar una metodología instruccional transmisiva o una constructivista. Omnia 2011; 17: 23-34.

13. Ramírez I, Maldonado C, Villacorta R, Gallardo G. Estudio sobre actitudes e intereses científicos en bachilleres de los colegios Sagrado Corazón de Jesús y San Agustín. Revista Ventana Científica 2016; 7: 1-8.

14. Pozo J, Gómez-Crespo MA. Aprender y enseñar ciencia. Del conocimiento cotidiano al conocimiento científico. Madrid: Morata; 1998.

15. Carrillo-Larco M, Carnero A. Autoevaluación de habilidades investigativas e intención de dedicarse a la investigación en estudiantes de primer año de medicina de una universidad privada en Lima, Perú. Revista Médica Herediana 2013; 24 : $17-25$.

16. Vázquez A, Manassero MA. El descenso de las actitudes hacia la ciencia de chicos y chicas en la educación obligatoria. Ciência \& Educação (Bauru) 2011; 17: 249-68.

17. Jenkins W, Nelson W. Important but not for me: students' attitudes towards secondary school science. Research in Science \& Technological Education 2005; 23: 41-57.

18. Vázquez A, Acevedo JA, Manassero MA. Más allá de una enseñanza de las ciencias para científicos: hacia una educación científica humanística. Revista Electrónica de Enseñanza de las Ciencias 2005. URL: https//www.saum.uvigo.es/reec/ volume-nes/volumen4/ART5_Vol4_N2.pdf.

19. Plazas M, Gómez M, Castro C. Actitud en estudiantes de ciencias de la salud hacia el conocimiento científico. Revista Ciencias de la Salud 2013; 11: 83-91.

20. Domínguez S. Campos de significación de la actividad científica en estudiantes universitarios. Perfiles Educativos 2013; 35: 28-47.

21. Díaz-Vélez C, Manrique L, Galán E, Apolaya S. Conocimientos, actitudes y prácticas en investigación de los estudiantes de pregrado de facultades de medicina del Perú. Acta Médica Peruana 2008; 25: 9-15.

22. Dirección de Ciencia y Tecnología. Catálogo de Proyectos de Investigación. Ganadores de Exposición y Jornadas Científicas. Versión I. Sucre: Universidad Mayor, Real y Pontificia de San Francisco Xavier de Chuquisaca; 2015.

23. CEUB-UMSA. Memoria de la feria nacional universitaria de investigación ciencia y tecnología, Buenas+Ideas. La Paz: Sistema de la Universidad Boliviana; 2015.

24. CEUB-UMRP SFXCH. Memoria de la feria nacional universitaria de investigación ciencia y tecnología, Buenas +Ideas. Sucre: Sistema de la Universidad Boliviana; 2016.

25. Cabrera JA, Cruzado C, Purizaca N, López R, Lajo Y, Peña E, et al. Factores asociados con el nivel de conocimientos y la actitud hacia la investigación en estudiantes de medicina en Perú, 2011. Revista Panamericana de Salud Pública 2013; 33 166-73.

26. De la Cruz C. Actitudes hacia la investigación científica en estudiantes universitarios: análisis en dos universidades nacionales de Lima. Psique Mag 2013; 2: 1-16. 